\title{
Incidental finding of isolated double orifice mitral valve in a patient with severe mitral stenosis related to radiotherapy
}

\author{
Alex GT dos Santos ${ }^{1}$, Iana F Prates ${ }^{1}$, Rodrigo B Silva ${ }^{1}$, Eline Lobo ${ }^{2}$, Luiz AP de Souza ${ }^{1,2}$ and Rafael M Fernandes ${ }^{1,2 *}$ \\ ${ }^{1}$ Bahiana School of Medicine and Public Health, Salvador - BA, Brazil \\ ${ }^{2}$ ADS Clinic, Brazil
}

\section{Introduction}

The Double Orifice Mitral Valve (DOMV) is an extremely rare condition, accounting for about $1 \%$ of congenital heart disease, and is caused by insufficient embryonic fusion of endocardial cushions $[1,2]$. Because it is a congenital disease, in the great majority of cases, it is detected in childhood and associated with other malformations [2]. However, there are reports in the literature of mitral stenosis associated with two mitral orifices (pseudo-DOMV) because of an acquired disease, rheumatic valvopathy for example, and differs from the DOMV due to the absence of independents tensor apparatus attached to each mitral valve orifice [3].

Another acquired valvopathy, whose incidence has increased significantly, is the valvopathy caused by radiotherapy [4]. However, pseudo-DOMV related to radiotherapy is a complication not well described in the literature yet. We present the case of a patient undergoing radiotherapy who, after the end of treatment, had severe mitral stenosis associated with two mitral orifices.

\section{Case report}

In this case report, we present a 76-year-old female patient, without cardiovascular comorbidities, who was diagnosed with invasive ductal carcinoma of the left breast (clinical stage IIIC) in 2016. Right after the initial diagnosis, she underwent total mastectomy and started adjuvant chemotherapy with Adriamycin and cyclophosphamide every 21 days for 2 months. After a month of interval, the patient gave sequence with paclitaxel weekly for about 3 months. And a month after the end of chemotherapy, the radiotherapy treatment started for another month (5000cGy).

Four months before the beginning of the chemotherapy treatment, an echocardiogram was performed, which showed only a slight thickening of the mitral valve, but there were no significant morphofunctional changes, being described as open and preserved mobility and without DOMV. Two months after the end of the radiotherapy treatment and 10 months after the start of the chemotherapy treatment, a new echocardiogram was performed, and demonstrated an important alteration of the mitral valve anatomy, which became fibrocalcified and evolved by mild to moderate stenosis. However, the description of absence of DOMV in the valve was maintained. Due to this rapid and significant pre and post treatment evolution, a new echocardiogram was requested for a more complete evaluation of the patient's condition. In this new examination, the presence of a mitral valve with thickened cusps combined with significant calcification, and the presence of a double orifice valve opening associated to severe mitral stenosis (mean diastolic mitral flow gradient $=14 \mathrm{mmHg}$ and $\mathrm{VA}=0.9 \mathrm{~cm}^{2}$ by planimetry) was evidenced (Figure 1 ).
Due to the confirmation of the rapid progression of valvular disease, cardiac magnetic resonance imaging (MRI) was requests to confirm the diagnosis, which demonstrated the presence of severe mitral stenosis associated with two orifices and absence of independent subvalvular apparatus (Figure 2).

\section{Discussion}

The double orifice mitral valve (DOMV) is a rare condition associated to congenital causes and it is often followed by other cardiac abnormalities [5]. The presence of tensor apparatus independently biding to the two valvar orifices represents an important characteristic of this condition and this mark differentiate the DOMV by other similar valvar lesions [6]. Considering that it is a congenital condition and it is accompanied by other cardiac abnormalities the diagnosis is done in the childhood. Therefore, it is rare to diagnose this condition in the adult life. The DOMV acquired is extremely uncommon and there are a few numbers of reported cases in literature. One of these cases shows an elderly patient presented with valvar thickening associated to the leaflet fusion. Its condition was described as pseudo DOMV. In

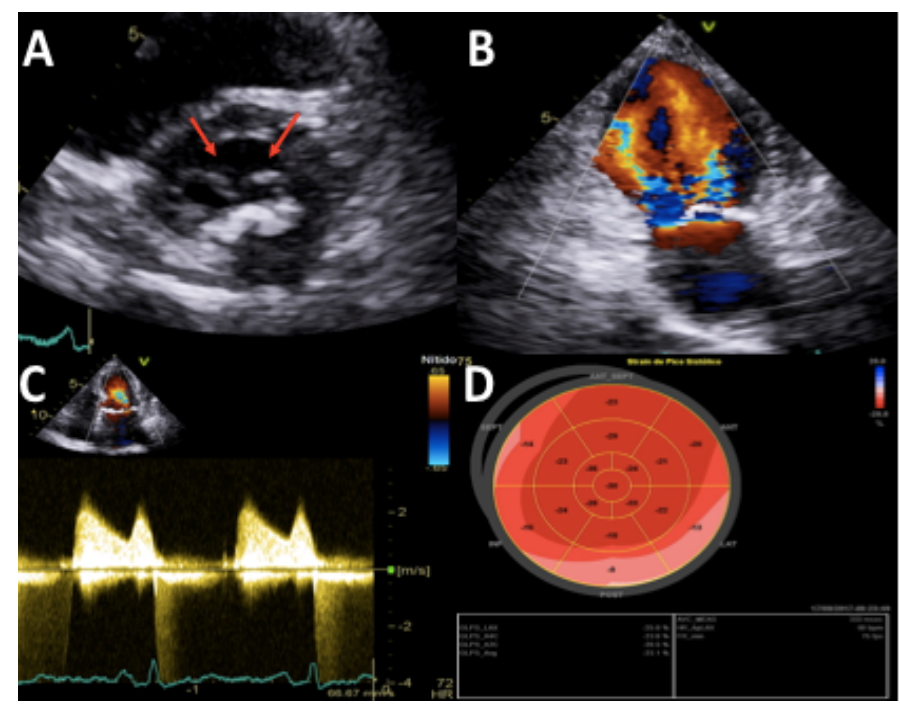

Figure 1. Chest X-ray with a severe cardiomegaly caused by biatrial dilatation

${ }^{*}$ Correspondence to: Rafael Modesto Fernandes, Bahiana School of Medicine and Public Health, Dom João VI Avenue, 275, Brotas, Salvador, Bahia, Brazil, Tel: +557199901-5141/+55713276-8200; E-mail: rafaelmodesto@usp.br

Received: May 04, 2019; Accepted: May 14, 2019; Published: May 17, 2019 


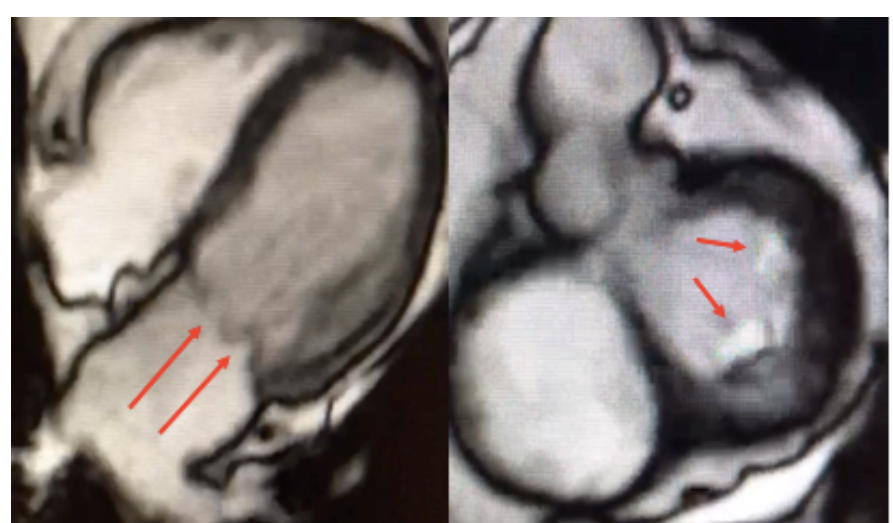

Figure 2. A and B. Incidences of cardiac MRI showing double mitral orifice with severe stenosis according to the mitral valve area.

other words, it was a valvulopathy with double orifice and absence of independent tensor apparatus [3].

This case describes a patient without previous cardiac abnormalities who presented a severe mitral stenosis in a short period after radiotherapy. Valvulopathy secondary to radiotherapy is well described in literature. However, it presents a long period to the development of symptoms, as about 12 to 16 years [7]. In this case, the echocardiogram before the onset of the treatment did not showed significant abnormalities. However, a new exam after the treatment demonstrated severe mitral stenosis associated to DOMV. The most common lesions secondary to radiotherapy are fibrosis and focal valvar calcification. DOMV is still not reported in the literature [8,9].

The main hypotheses to this case was: (1) A pre-existing structural disease (rheumatic disease or previous DOMV not identified) that presented an accelerated progression after radiotherapy or (2) a pseudo
DOMV secondary to radiotherapy. The brief period of evolution contributes to the first hypothesis. However, considering that the MRI and the echocardiogram did not show independent tensor apparatus, the patient did not present suggestive characteristics of rheumatic disease and, finally, the absence of structural abnormalities we concluded that it was a pseudo DOMV secondary to radiotherapy.

\section{Conflicts of Interest}

The authors declare that there is no conflict of interest.

\section{References}

1. Zalzstein E, Hamilton R, Zucker N, Gross GJ, Levitas A (2004) Presentation, Natural History, and Outcome in Children and Adolescents with Double Orifice Mitral Valve. Am J Cardiol 93: 1067-1069. [Crossref]

2. Liu S, Ren W, Ma C, Yang J (2017) Congenital Double-Orifice Mitral Valve in Asymptomatic Patients. Int Hear J Assoc 59: 213-215. [Crossref]

3. Arunkumar P, Rajesh T (2016) Pseudo-double orifice mitral valve or heart in heart mitral valve. J Indian Coll Cardiol.

4. Yusuf SW, Sami S, Daher IN (2011) Radiation-Induced Heart Disease: A Clinical Update. Cardiol Res Pract 2011: 317659. [Crossref]

5. Rowe DW, Desai B, Bezmalinovic Z, Dessai JM, Wessel RJ, et al. (1984) TwoDimensional Echocardiography in Double Orifice Mitral Valve. Am Coll Cardiol 4 429-33. [Crossref]

6. Das BB, Pauliks LB, Knudson OA, Kirby S, Chan K, et al. (2002) Double-Orifice Mitral Valve with Intact Atrioventricular Septum: An Echocardiographic Study with Anatomic and Functional Considerations. Am Soc Echocardiogr 18: 231-236. [Crossref]

7. Carlson RG, Mayfield WR, Normann S, Alexander JA (1991) Radiation-Associated Valvular Disease. Chest 99: 538-45. [Crossref]

8. Brand M, Abadi C, Aurigemma G, Dauerman H, Meyer T (2001) Radiation-associated valvular heart disease in Hodgkin's disease is associated with characteristic thickening and fibrosis of the aortic-mitral curtain. J Heart Valve Dis 10: 681-685. [Crossref]

9. Katz NM, Hall AW, Cerqueira MD (2001) Radiation induced valvulitis with late leafle rupture. Heart 86: e20 [Crossref]

Copyright: (C2019 GT dos Santos A. This is an open-access article distributed under the terms of the Creative Commons Attribution License, which permits unrestricted use, distribution, and reproduction in any medium, provided the original author and source are credited. 